\title{
Well-defined lignin model films from colloidal
}

\section{lignin particles}

Muhammad Farooq*a, Zou Tao ${ }^{a}$, Juan José Valle-Delgado ${ }^{a}$, Mika Henrikki Sipponen ${ }^{b}$, Maria Morits ${ }^{a}$, Monika Österberg* ${ }^{a}$

a Aalto University, School of Chemical Engineering, Department of Bioproducts and Biosystems, Vuorimiehentie 1, 02150 Espoo, Finland

b Department of Materials and Environmental Chemistry, Stockholm University, Stockholm, 10691, Sweden.

*Corresponding authors: monika.osterberg@aalto.fi,+358505497218 


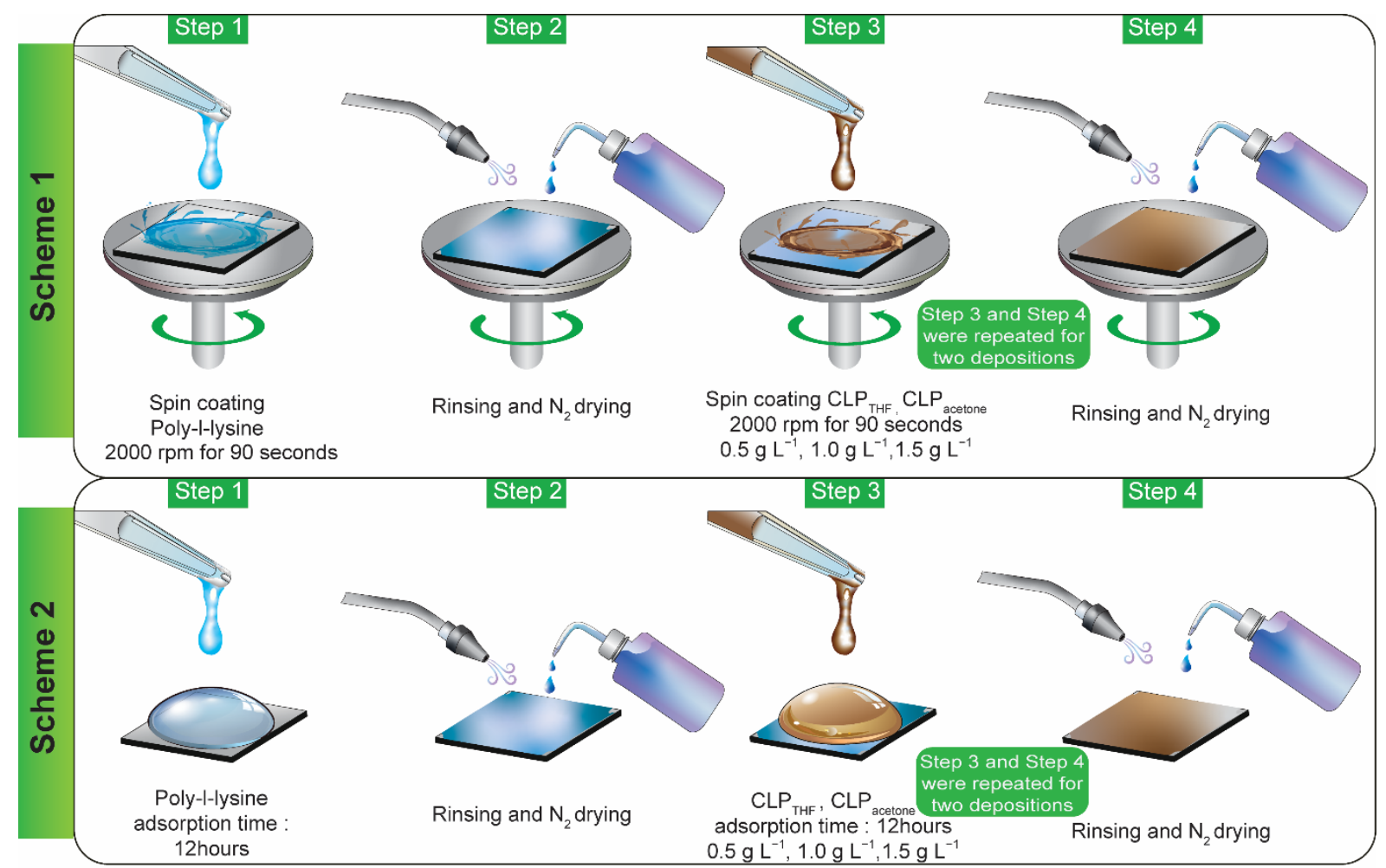

Scheme S1. Schematic illustration of thin films preparation. Both Scheme 1 and Scheme 2 where used for surface coverage analysis. For WCA measurements and QCM-D experiments, Scheme 2 was employed utilizing both CLPTHF and CLPacetone only at $1.5 \mathrm{~g} \mathrm{~L}^{-1}$ concentration and adsorption times of $30 \mathrm{~min}$ and $60 \mathrm{~min}$ for PLL and CLPs. Silica wafers were used as substrates for WCA measurements and gold sensors for QCM-D experiments.

Table S1. Comparison of surface coverage of thin films

\begin{tabular}{|c|c|c|c|c|c|c|}
\hline & \multirow[b]{2}{*}{$\begin{array}{c}\text { Thin film preparation } \\
\text { method }\end{array}$} & \multirow{2}{*}{$\begin{array}{c}\text { Concentration } \\
\mathrm{g} \mathrm{L}^{-1}\end{array}$} & \multicolumn{2}{|c|}{ Single deposition } & \multicolumn{2}{|c|}{ Two depositions } \\
\hline & & & $\begin{array}{c}\text { Surface } \\
\text { coverage } \\
(\%)\end{array}$ & STD & $\begin{array}{c}\text { Surface } \\
\text { coverage } \\
(\%)\end{array}$ & STD \\
\hline \multirow{6}{*}{$\underset{\theta}{0}$} & \multirow{3}{*}{ Adsorption } & 0.5 & 41.5 & 4.8 & 47.5 & 2.3 \\
\hline & & 1 & 63 & 2.7 & 53.4 & 8.1 \\
\hline & & 1.5 & 67.7 & 1.1 & 82.2 & 2.1 \\
\hline & \multirow{3}{*}{ Spin coating } & 0.5 & 6.7 & 2.5 & 35.7 & 2.1 \\
\hline & & 1 & N/A & N/A & 49.4 & 1.5 \\
\hline & & 1.5 & 20.5 & 2.4 & 64 & 2.8 \\
\hline \multirow{6}{*}{ تِ } & \multirow{3}{*}{ Adsorption } & 0.5 & 64.9 & 0.4 & 46.9 & 0.1 \\
\hline & & 1 & 54.5 & 0.8 & 61.8 & 1.5 \\
\hline & & 1.5 & 71.4 & 0.2 & 72.5 & 0.8 \\
\hline & \multirow{3}{*}{ Spin coating } & 0.5 & 10.6 & 1.3 & 54.6 & 0.3 \\
\hline & & 1 & 15 & 0.7 & 67.3 & 0.6 \\
\hline & & 1.5 & 21.4 & 1.5 & 67.5 & 1.2 \\
\hline
\end{tabular}




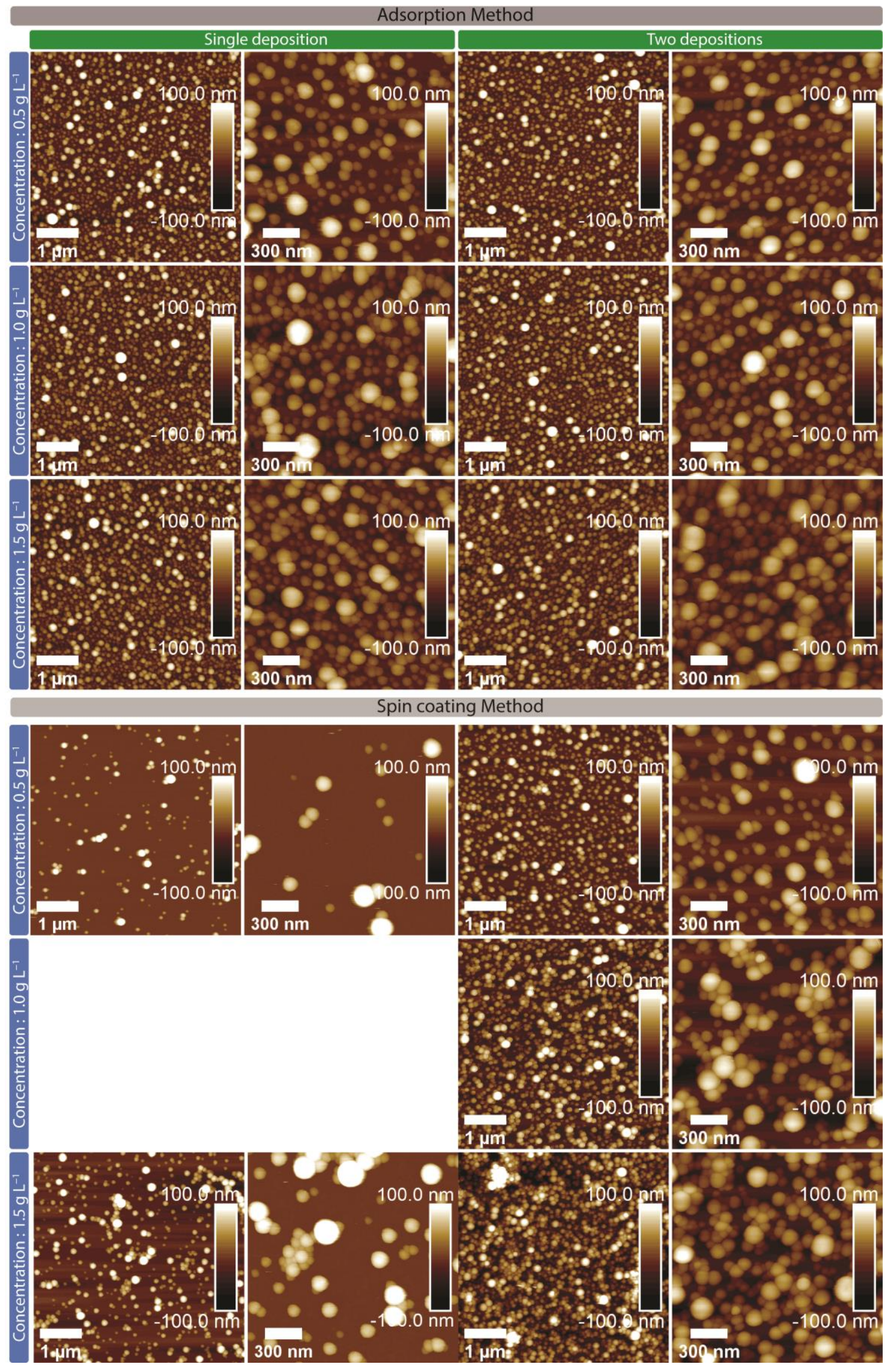

Figure S1. AFM topography images of the single and two depositions CLPTHF thin films 
prepared using spin coating and adsorption at $0.5 \mathrm{~g} \mathrm{~L}^{-1}, 1.0 \mathrm{~g} \mathrm{~L}^{-1}, 1.5 \mathrm{~g} \mathrm{~L}^{-1}$ dispersion concentrations
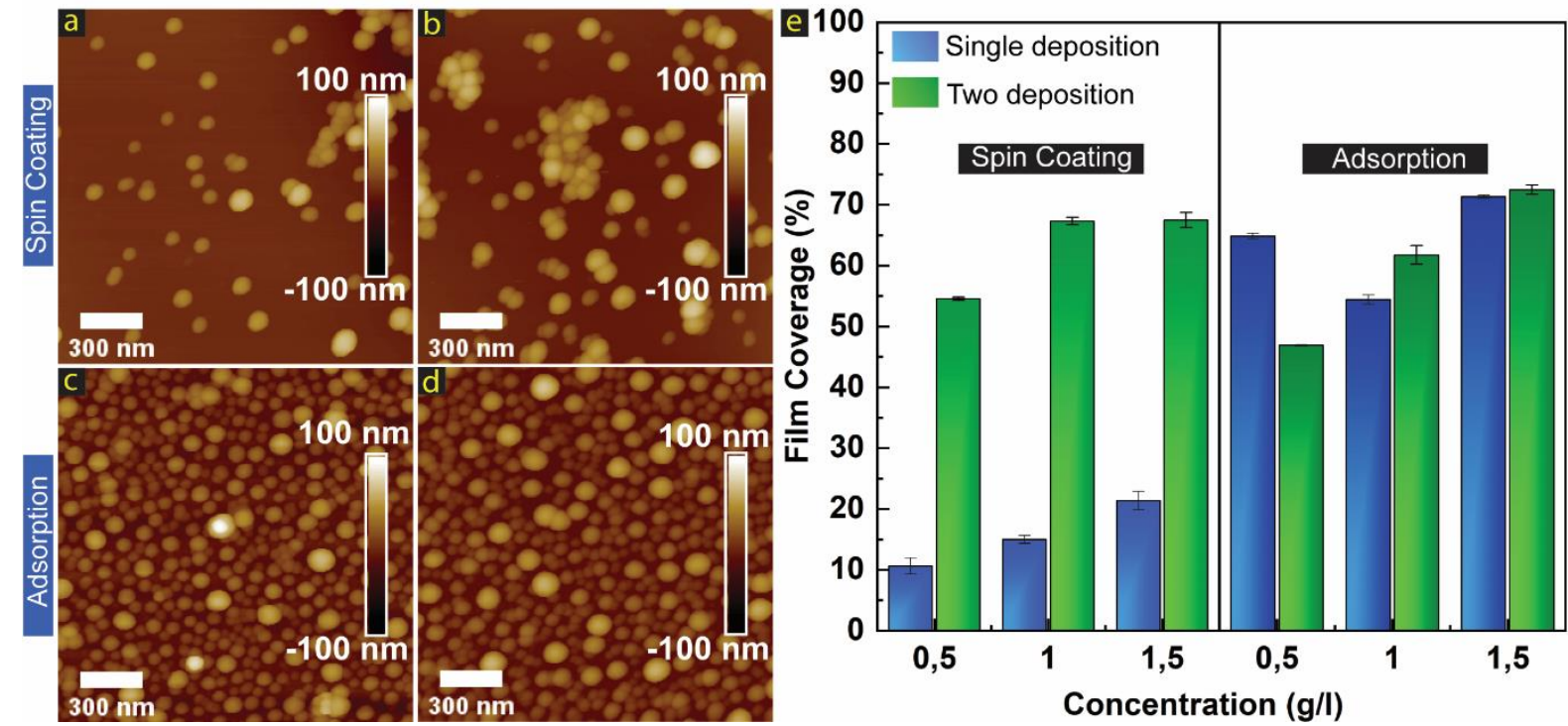

Figure S2. Effect of deposition method and CLP concentration on surface coverage of CLP acetone thin films prepared on silica wafers. AFM topography images of the single deposition of CLPacetone thin films prepared using spin coating at (a) $0.5 \mathrm{~g} \mathrm{~L}^{-1}$ and (b) $1.5 \mathrm{~g} \mathrm{~L}^{-1}$, and by adsorption method at (c) $0.5 \mathrm{~g} \mathrm{~L}^{-1}$ and (d) $1.5 \mathrm{~g} \mathrm{~L}^{-1}$ concentration (12 hours of adsorption time for each adsorbed layer). (e) Comparison of surface coverage of CLP acetone thin films prepared by spin coating and adsorption methods. All AFM topography images are $1.7 \mu \mathrm{m} \times 1.7 \mu \mathrm{m}$ in size. 


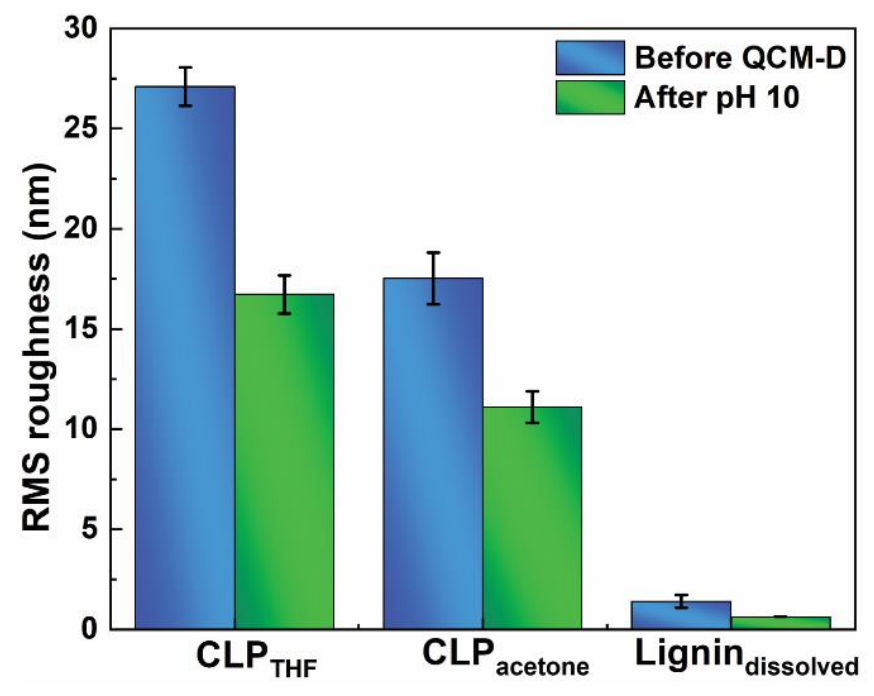

Figure S3. Change in the root mean squared (RMS) roughness $(\mathrm{Rq})$ of CLP acetone, CLPTHF and Lignin $_{\text {dissolved }}$ thin films after exposure to $\mathrm{pH} 10$.
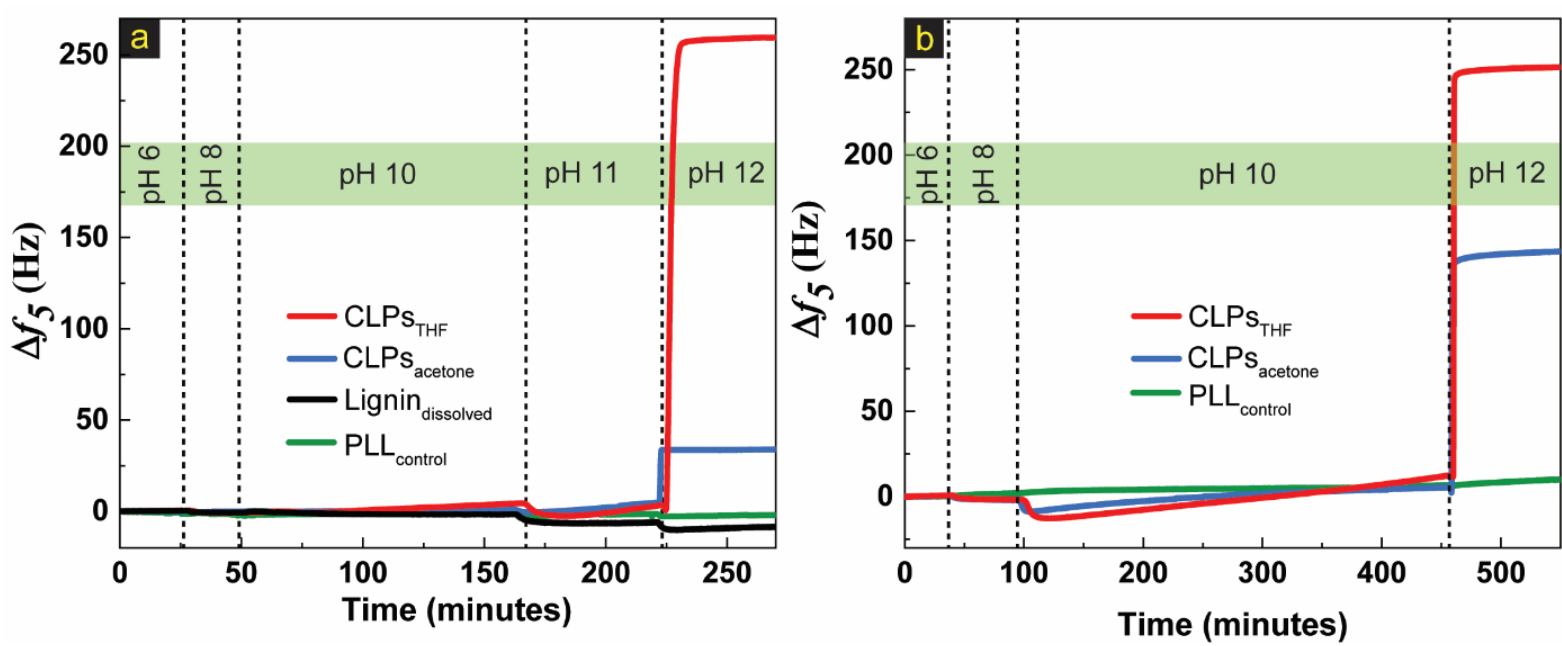

Figure S4. Replicate QCM-D experiments depicting change in frequency, as the $\mathrm{pH}$ was incrementally alkalized for CLP acetone, CLPTHF and Lignindissolved thin films 


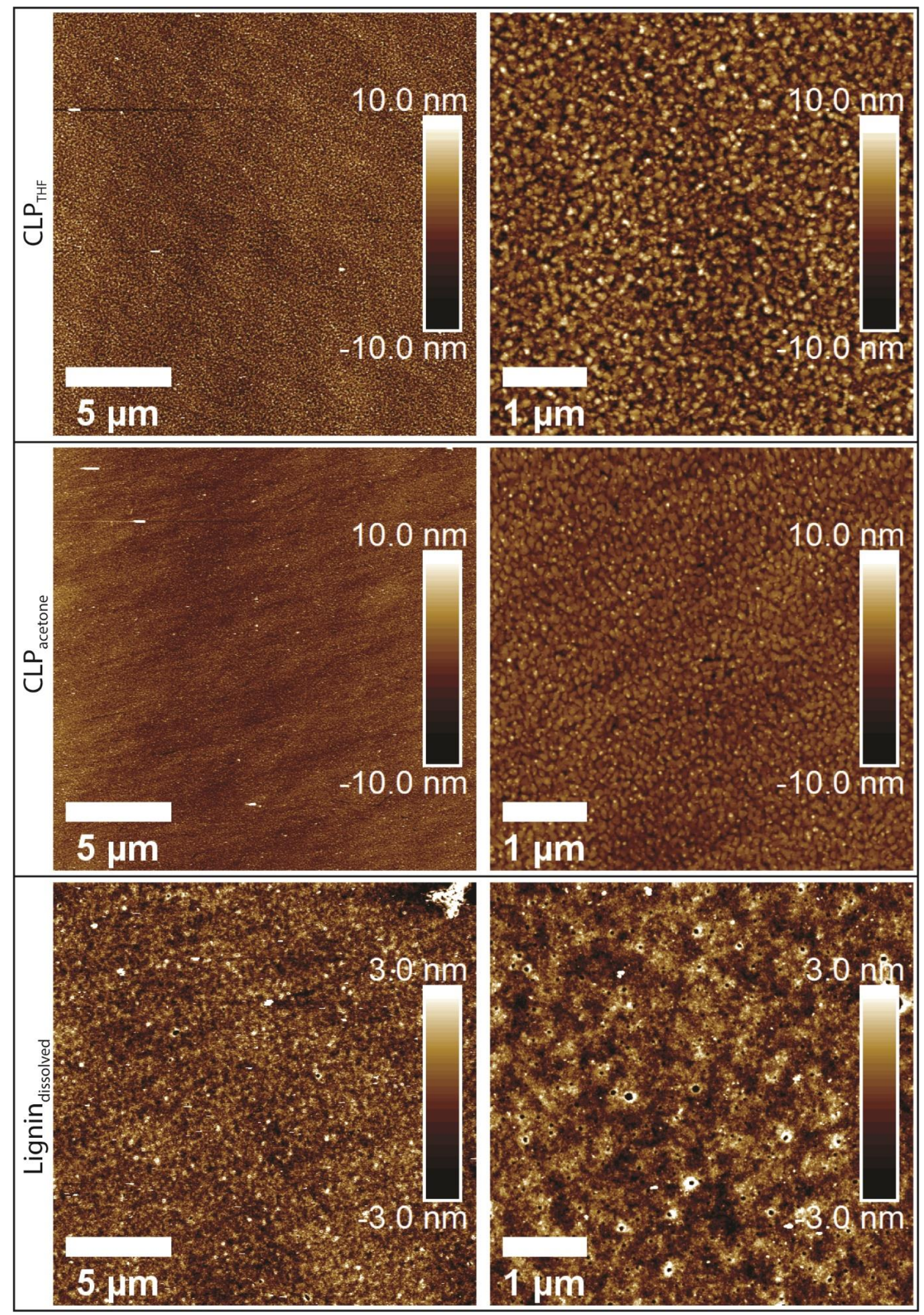

Figure S5. AFM height images of CLP acetone, $_{\text {CLP }}$ THF $_{\text {and }}$ Lignin dissolved $_{\text {thin }}$ films respectively after exposure at $\mathrm{pH} 12$ 